\title{
Mesenchymal stem cells of human placenta and umbilical cord suppress T-cell proliferation at G0 phase of cell cycle
}

\begin{abstract}
Mesenchymal stem cells (MSC) generated from human umbilical cord (UC-MSC) and placenta (PLC-MSC) were assessed and compared for their immunomodulatory function on $\mathrm{T}$ cells proliferation by analysis of the cell cycle. Mitogen stimulated or resting T cells were co-cultured in the presence or absence of MSC. T-cell proliferation was assessed by tritiated thymidine ((3) $\mathrm{H}-\mathrm{TdR}$ ) assay and the mechanism of inhibition was examined bycell cycle and apoptosis assay. Both UC-MSC and PLC-MSC profoundly inhibited the proliferation of T-cell, mainly via cellto-cell contact. MSC-mediated anti-proliferation does not lead to apoptosis, but prevented T cells from entering $S$ phase and they therefore accumulated in the $G(0) / G(1)$ phases. The antiproliferative activity of MSC was related to this cell cycle arrest of T-cell. UC-MSC produced a greater inhibition than PLC-MSC in all measured parameters.
\end{abstract}

Keyword: Mesenchymal stem cell; Cell cycle arrest; Umbilical cord; Placenta; T cells 